\title{
PERFORMANCES OF MULTI-FREQUENCY VOLTAGE TO CURRENT CONVERTERS FOR BIOIMPEDANCE SPECTROSCOPY
}

\author{
Shekh Md Mahmudul Islam*, Mohammad Anisur Rahman Reza and Md Adnan Kiber \\ Department of Applied Physics, Electronics and Communication Engineering, \\ University Of Dhaka, Dhaka-1000, Bangladesh \\ *Email: mahmud_apececadet@yahoo.com (corresponding author)
}

\begin{abstract}
Breast cancer is one of the most common diseases affecting women and the mortality rate is very high in Bangladesh, mainly due to the lack of early diagnosis. X-ray mammography is not available widely in Bangladesh, besides it has an associated biological hazard. Multi-frequency bio-impedance measurement or bio-impedance spectroscopy has a great potential in many biomedical applications including breast cancer screening which involves tissue characterization. In Bio-impedance spectroscopy a range of frequencies of low level current is injected to the human body and corresponding bio-impedance is measured. In a typical bio-impedance measurement system an ac source with a constant current amplitude is an essential part in which the current amplitude should not change with frequency or load, the latter being typically of the order of a few kilo ohms in the human body. The constant current source is usually obtained from a voltage-to-current converter and several standard designs are available, for example a floating current source, a Howland current source and an enhanced Howland current source. The present work was taken up to perform a comparative study of these three types of current sources based on a commonly available low cost operational amplifier, 741. It was found that the enhanced Howland current source gave the best performance, the current being almost constant up to a frequency of about $10 \mathrm{kHz}$ for a load of $10 \mathrm{k} \Omega$. This work gives an understanding of this fundamental circuit segment that is essential for the development of a Bioimpedance Spectroscopy system.
\end{abstract}

Keywords: Voltage-to-current converter, Bioimpedance Spectroscopy, Multi-frequency Impedance

\section{INTRODUCTION}

Worldwide, breast cancer is now one of the most common cancer and is the leading cause of deaths from cancer among women with approximately 1.3 million new cases and an estimated 4, 58,000 death reported in the year of 2011[1]. One in every eight women in the USA has a chance of having invasive breast cancer during her lifetime. The risk of breast cancer increases with age. As there is no cancer registry system in Bangladesh, so we do not have statistics on this. Developing countries especially Bangladesh is in a high risk condition in breast cancer.

The most common form of screening system available for breast cancer detection is Mammography. Mammography is the process of using $\mathrm{x}$-ray to examine the human breast and is used as a diagnostic and screening tool. Although this uses low energy x-rays, any ionizing radiation is harmful for human. Besides, the number of mammography equipment available in Bangladesh is very small, and the cost of the test is high. Attempts are on at developing multi-frequency bio-impedance spectroscopy as an alternative to this screening procedure $[2,3,4]$ as it is non-invasive, harmless to human body and potentially cheap. If found positive in the screening test the patient may be referred to the more accurate tests and expensive medical imaging method like Mammogram, MRI etc.

Bio-impedance is a term used to describe the response of a living organism to an externally applied electric current. It is a measure of the opposition to the flow of that electric current through the tissues, the opposite of electrical conductivity. The measurement of the bio-impedance (or bioelectrical impedance) of the humans and animals has proved useful as a non-invasive method for measuring such parameters as blood flow and body composition (known as bioelectrical impedance analysis or simply BIA). 
The human body is made of tiny building blocks called cells. Cells consist of a group of organelles which are floating in dissolution enclosed within the plasma-membrane. The cell again is embedded in an electrolyte permeating throughout the body. Overall the electrical model may be taken to consist of capacitive and resistive elements as shown in Fig.1. Here $R_{m}$ and $C_{m}$ represent the Membrane impedance, $R_{\mathrm{e}}$ the external fluid resistance, and $R_{\mathrm{i}}$ the internal cell resistance. The yellow ellipse indicates the limit between the internal and external sides of the cell.

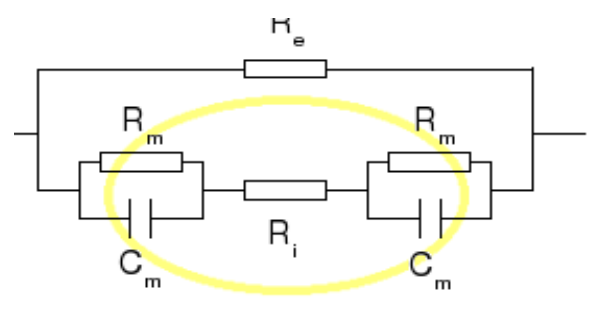

Fig. 1: Electrical model of a human cell.

Usually the body creates healthy, normal cells that divide and grow in a self-limiting way. If a cell divides and grows in an uncontrolled way a tumor or abnormal body cells grouped together in the form of a mass or lump can start to form and grow. The normal cells of a particular organ of the body possess specific impedance characteristics when subjected to electrical signals of varying frequencies. Since the cancerous tissues are different from normal healthy tissues, the electrical impedance characteristics are expected to be different. This behaviour can be utilized to detect any abnormality of the particular organ The breast cancer screening system is basically a spectroscopy system to exploit this nature to detect or screen any suspicious response. Typical impedance characteristics of normal and cancerous breast tissues are shown in Fig.2.

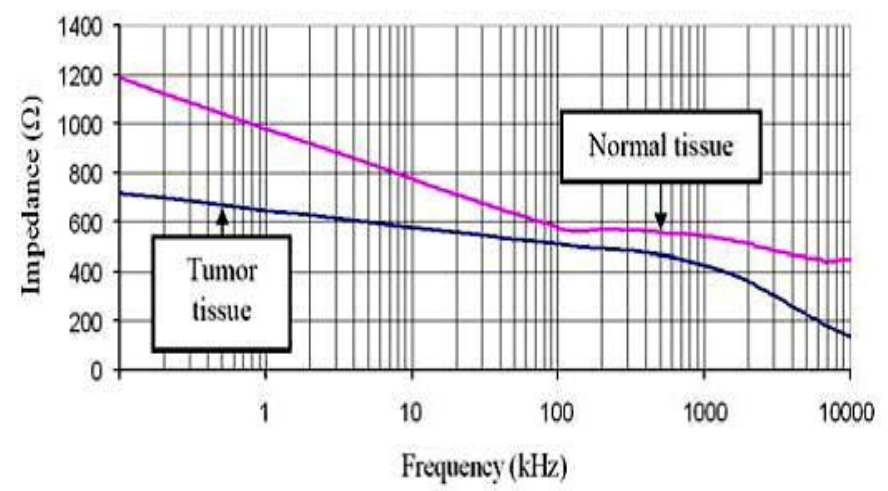

Fig.2: Frequency characteristics of the Impedance of normal and cancerous breast tissue

A bio-impedance spectroscopy system typically uses four electrode methods. Alternating currents of different frequencies are applied through two electrodes while the resulting voltages are measured across the other two electrodes. As most of the measurements are made on the surface of the body, this method can be described as non-invasive. Typically very low currents, of the order of $1 \mathrm{~mA}$, are applied at frequencies of $10 \mathrm{kHz}$ or higher. At these levels no harm to human tissue has been reported to date. For convenience in measurement the driven current is kept constant in most measurement methods involving four electrodes. The reliability of a multi-frequency bio-impedance spectroscopy system is largely dependent on the constancy of the driven current for typical changes in the impedance of body tissues. Change in bio-impedance of normal tissues compared to that of cancerous tissues can be found in a wide range of frequencies from $1 \mathrm{kHz}$ to $1 \mathrm{MHz}$, however, measurements up to $100 \mathrm{kHz}$ is adequate in breast cancer application as suggested by Fig.2. A bio-impedance system in cancer screening should be capable of delivering constant current to the patient over this range of frequencies. It is also 
important to note that the effective bio-impedance of various tissues or organs vary from normal to diseased case as well as from person to person. It is found that bio-impedance of typical human tissues varies from $1 \mathrm{~K} \Omega$ to $50 \mathrm{k} \Omega$ depending on the conditions. So a good bio-impedance spectroscopy system for breast cancer screening should provide a constant current over a range of frequencies from $1 \mathrm{KHz}$ to $100 \mathrm{KHz}$ and load impedances from $1 \mathrm{k} \Omega$ to $50 \mathrm{k} \Omega$. This paper will focus on measuring the performances of different voltage-to-current converters for meeting the desired requirements of a reliable Bio-impedance spectroscopy system.

\section{DESIGN OF CONSTANT CURRENT SOURCES AND EXPERIMENTAL METHODS}

A constant current source is usually designed in the form of a voltage-to-current converter. The other name of a voltage-to-current converter is voltage controlled current source (VCCS), as the output current is controlled by the input voltage. In the present work we have used three different types of voltage to current converters:

- Voltage-to-current converter with floating load.

- Basic Howland Voltage-to-current converter

- The Enhanced Howland Voltage-to-current Converter

Standard circuits for the above are available from different sources. In the present work we performed a comparative study of these circuits using a commonly available op-amp, 741. This IC has a unity gain bandwidth of $1 \mathrm{MHz}$ which will affect the performance at higher frequencies.

\subsection{Voltage-to-current converter with floating load:}

Fig. 3 shows a voltage-to-current converter in which load resistor $\mathrm{R}_{1}$ is floating (not connected to ground). The input voltage $v_{\text {in }}$ is applied at the non-inverting input terminal. This circuit is also called a current series negative feedback amplifier because the feedback voltage $v_{f}$ across $R_{2}$ (applied to the inverting terminal) depends on the output current $I_{0}$ through $R_{1}$ and is in series with the input difference voltage $\mathrm{V}_{\text {id. }}\left(=\mathrm{v}_{\text {in }}-\mathrm{v}_{\mathrm{f}}\right)$.

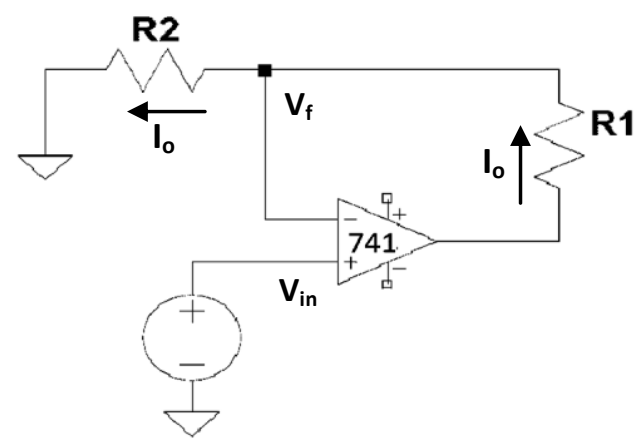

Fig.3: voltage-to-current converter with floating load

Writing Kirchhoff's voltage equation for the Input loop, $V_{\text {in }}=V_{\text {id }}+V_{f}$

Since the gain of the op-amp A is very large, $V_{\text {id }} \cong 0 \mathrm{~V}$. Therefore, $V_{\text {in }} \cong V_{f}$ which again equals $R_{2} I_{0}$.

Therefore, output current, $\quad I_{0}=V_{\text {in }} / R_{2}$.

For delivering $1 \mathrm{~mA}$ we chose $\mathrm{V}_{\text {in }}=5 \mathrm{~V}$, and $\mathrm{R}_{2}=5 \mathrm{k} \Omega$.

\subsection{Basic Howland Voltage-to-current converter:}


This voltage-to-current converter is shown in Fig.4. In this circuit, one terminal of the load $R_{L}$ is grounded and load current is controlled by an input voltage $V_{\text {in }}$. The analysis of this circuit is accomplished by first determining the voltage $\mathrm{V}_{1}$ at the non-inverting input terminal and then establishing the relationship between $\mathrm{V}_{1}$ and the load current.

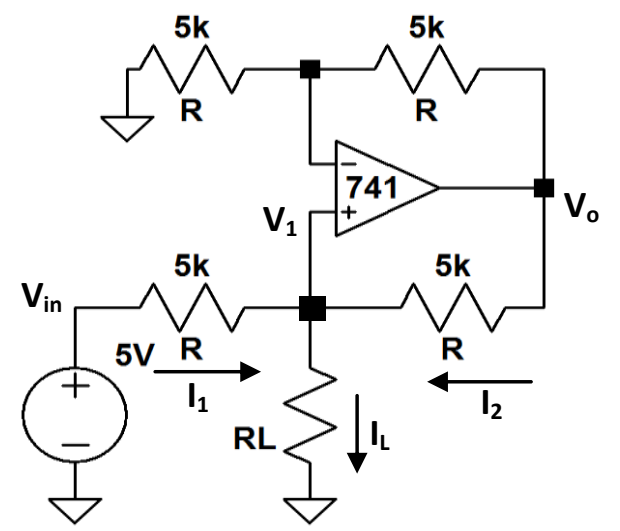

Fig.4: Basic Howland voltage-to-current converter

Writing Kirchhoff's current equation at non-inverting terminal,

$I_{1}+I_{2}=I_{L}$, from which we can write,

$\mathrm{I}_{1} \mathrm{R}+\mathrm{I}_{2} \mathrm{R}=\mathrm{I}_{\mathrm{L}} \mathrm{R}$

Or, $\quad\left(V_{\text {in }}-V_{1}\right)+\left(V_{o}-V_{1}\right)=I_{L} R, \quad$ i.e., $\quad V_{\text {in }}+V_{o}-2 V_{1}=I_{L} R$

Since the op-amp is connected in the non-inverting mode, the output voltage is

$$
\mathrm{V}_{\mathrm{o}}=(1+\mathrm{R} / \mathrm{R}) \mathrm{V}_{1}=2 \mathrm{~V}_{1}=\mathrm{V}_{\text {in }}+\mathrm{V}_{\mathrm{o}}-\mathrm{I}_{\mathrm{L}} \mathrm{R}
$$

Therefore, $\quad V_{\text {in }}=I_{L} R, \quad$ i.e., $\quad I_{L}=V_{\text {in }} / R$

This means that the load current depends on the input voltage $V_{\text {in }}$ and resistor $R$, and not on the load resistance $\mathrm{R}_{\mathrm{L}}$.

For delivering $1 \mathrm{~mA}$ current we used $\mathrm{V}_{\text {in }}=5 \mathrm{~V}$, and $\mathrm{R}=5 \mathrm{k} \Omega$.

\subsection{The Enhanced Howland Voltage-to-current converter:}

The structure of the enhanced Howland circuit is shown in Fig.5.

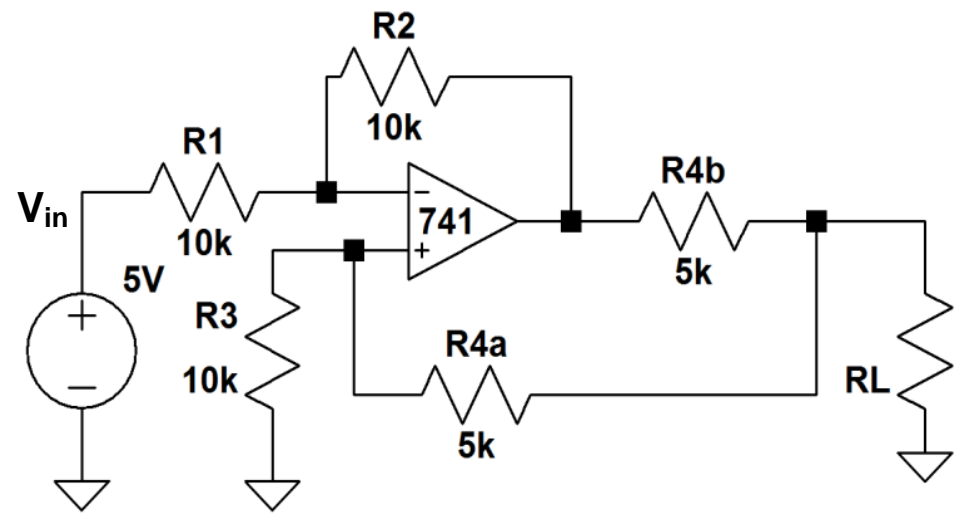

Fig. 5: Enhanced Howland Voltage-to-current Converter 
Its analysis is available in text books [5-7] and the output current $I_{0}$ is given in terms of input voltage $V_{\text {in }}$ as,

$$
I_{o}=\frac{V_{\text {in }} R_{2}}{R_{4 b} R_{1}}
$$

For the present work we used $\mathrm{V}_{\mathrm{in}}=5 \mathrm{~V}, \mathrm{R}_{4 \mathrm{~b}}=5 \mathrm{k} \Omega, \mathrm{R}_{2}=10 \mathrm{~K} \Omega, \mathrm{R}_{1}=10 \mathrm{~K} \Omega$ giving $\mathrm{I}_{\mathrm{o}}=1 \mathrm{~mA}$.

\section{RESULTS AND OBSERVATIONS}

The output currents for the three different types of voltage-to-current converters as obtained through experimentation using a 741 op-amp are shown in Fig. 6 for different frequencies and different load resistances.
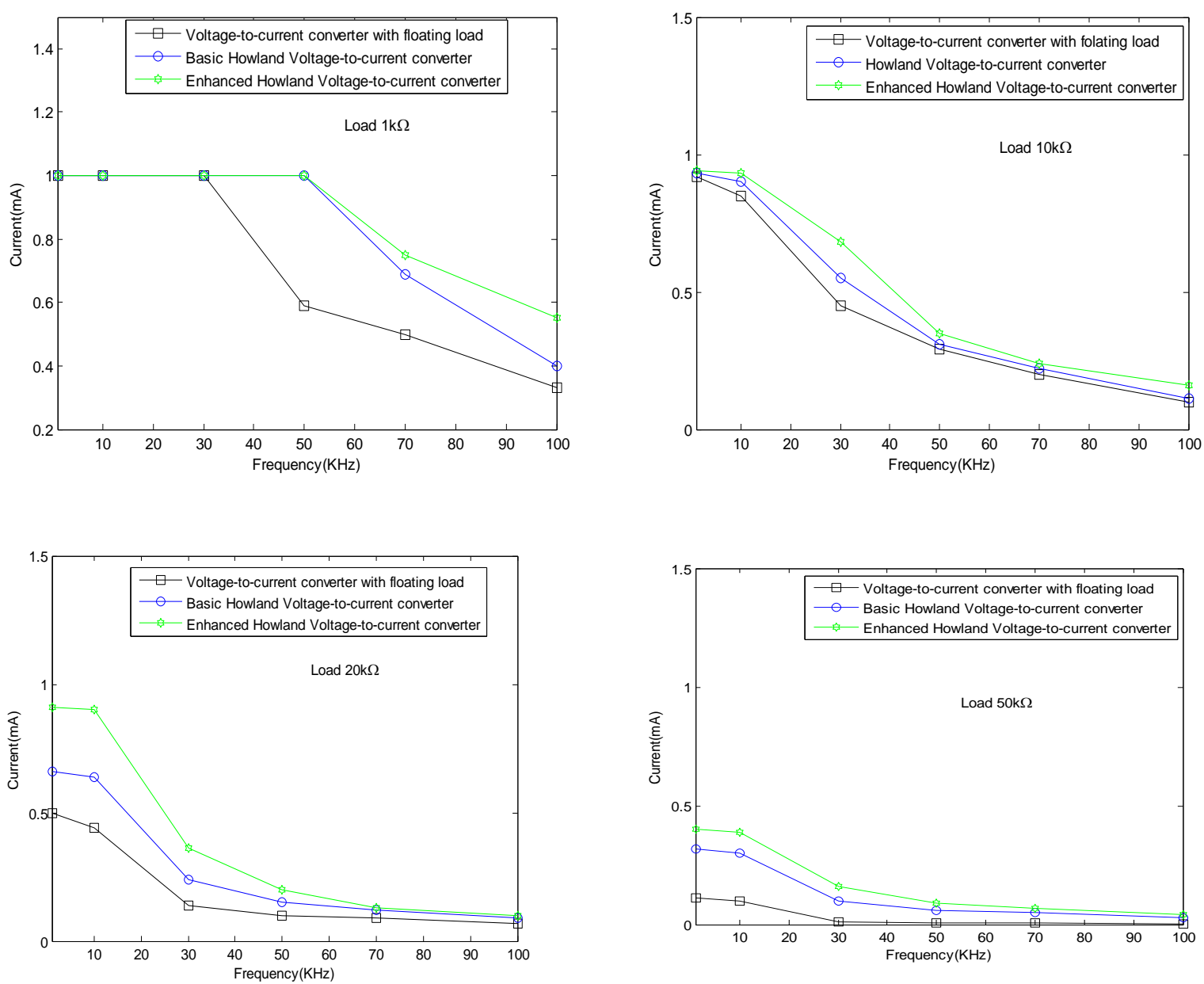

Fig.6: Comparative performance of the three types of voltage to current converters at different frequencies and for different loads.

The current is constant at the designed value of $1 \mathrm{~mA}$ up to $50 \mathrm{kHz}$ for $1 \mathrm{k} \Omega$ load for both basic Howland and enhanced Howland current sources. For higher loads the current levels are lower and are maintained reasonably constant up to about $10 \mathrm{kHz}$ only. The limited bandwidth of 741 is possibly responsible for the unsatisfactory performance of the experimental circuits. However, between the three types of circuits, the enhanced Howland current appears to perform the best. 


\section{CONCLUSION}

Multi-frequency Bio-impedance spectroscopy systems seem to be promising in early breast cancer screening procedure. One of the important subsystems of this system is a voltage-to-current converter which needs to give a constant current amplitude between $1 \mathrm{kHz}$ and $100 \mathrm{kHz}$ at least. In our research work we have designed and tested three different types of voltage-to-current converters. It is found that the enhanced Howland circuit has better performance than other two types. However, it is also clear that the type of op-amp IC is very important. The op-amp IC 741 used in the present study is not suitable for such a bioimpedance spectroscopy system. One needs an op-amp with much greater bandwidth.

\section{REFERENCES}

1. The New-York Times, Breast Cancer and its detection techniques in USA, March 2011.

2. Y.Zou,Z Guo,2002,A review of electrical impedance techniques for breast cancer detection, Medical Engineering \& Physics 25,79-90, 2003.

3. M A Kadir et al, Breast tumour characterization using dual frequency FIM, Abstracts, International Science Seminar Souvenir, Asiatic Society of Bangladesh, 2011

4. D.S Holder, Electrical impedance Tomography methods, History and applications, Institute of Physics publishing, 2005.

5. S Franco, Design with operational amplifiers and analog integrated circuits, 3rd edition, New York, McGraw-Hill, 2002.

6. Rezaul Karim Mazumder, Principles of Electronic Circuits, 1st edition, Systech Publication. 2006.

7. Robert F. Coughlin, Operational amplifiers and linear integrated circuits, 6th edition, Prentice Hall Electronics, 2006.

8. Ramakant A. Gayakward, Op-amps and linear integrated circuits, fourth edition, prentice hall of India, 2010-2011. 